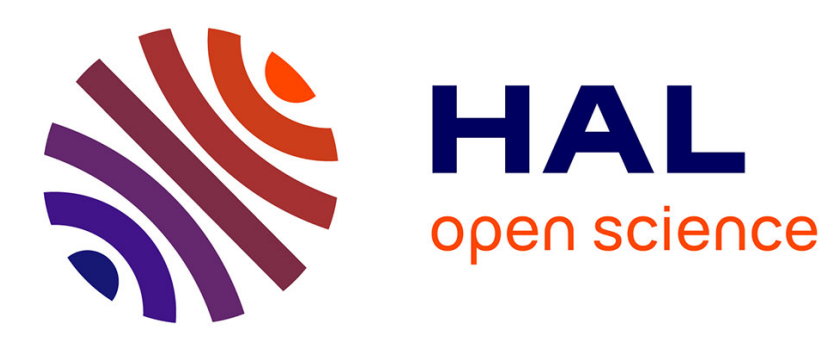

\title{
On the use of CALPHAD-based enthalpy-temperature relations in suboxidized corium plane front solidification modelling
}

\author{
V. Tiwari, R. Le Tellier
}

\section{- To cite this version:}

V. Tiwari, R. Le Tellier. On the use of CALPHAD-based enthalpy-temperature relations in suboxidized corium plane front solidification modelling. The $8^{\text {th }}$ European Review Meeting on Severe Accident Research - ERMSAR-2017, May 2017, Varsovie, Poland. cea-02434529

HAL Id: cea-02434529

https://hal-cea.archives-ouvertes.fr/cea-02434529

Submitted on 10 Jan 2020

HAL is a multi-disciplinary open access archive for the deposit and dissemination of scientific research documents, whether they are published or not. The documents may come from teaching and research institutions in France or abroad, or from public or private research centers.
L'archive ouverte pluridisciplinaire HAL, est destinée au dépôt et à la diffusion de documents scientifiques de niveau recherche, publiés ou non, émanant des établissements d'enseignement et de recherche français ou étrangers, des laboratoires publics ou privés. 


\title{
On the use of CALPHAD-based enthalpy-temperature relations in suboxidized corium plane front solidification modelling
}

\author{
V. Tiwari ${ }^{\mathrm{a}}$, R. Le Tellier ${ }^{\mathrm{a}, *}$ \\ ${ }^{a}$ CEA, DEN, DTN/SMTA/LPMA, Cadarache \\ F-13108 Saint Paul-lez-Durance, France
}

\begin{abstract}
The knowledge of in-vessel corium behaviour and associated risk of vessel failure are matters of prime interest within the framework of Severe Accident studies in a Light Water Reactor. Core meltdown during a severe accident results into formation of a corium pool at the bottom of the reactor vessel. This corium pool undergoes a density dependent liquid separation, thus exhibiting stratification into different phases. The distribution of phases depends on the time since the phase density depends on both temperature and composition of the phases. The evolution of stratification determines the impact of heat flux distribution at the lateral vessel wall, thereby determining the chances of success of the in-vessel retention strategy.

Within integral codes, the modelling of corium behaviour involves the coupling between lumped parameter thermal models and thermochemical models. Integral thermal models consist of mass and energy conservation equations that require inputs related to thermochemical properties of the materials, which are closely related to the state variables. In particular, the closure of energy conservation equations requires enthalpy-temperature relations. In the framework of multicomponent systems, the dependence of such relations to chemical composition is of importance and should be treated adequately to obtain a more accurate description of the phases depicted by the model. An approach to do so is to keep a general formulation of energy conservation equations in terms of specific enthalpies instead of substituting simplified enthalpy-temperature relations on a case-by-case basis in order to obtain models with an explicit temperature formulation. The enthalpy-temperature relation is then considered as an "Equation-of-State" (EOS) that can be written as: $\mathscr{H}: T,\left(w^{j}\right)_{j \in S} \rightarrow h$ along with its reciprocal relation $\mathscr{T}: h,\left(w^{j}\right)_{j \in S} \rightarrow T$ where $\left(w^{j}\right)_{j \in S}$ is the composition of the phase in terms of species mass fraction, $h$ the mass enthalpy and $T$ the temperature.

The present study seeks to explore the use of CALPHAD-based enthalpy temperature relations in the modelling of suboxidized in-vessel corium plane front solidification. Along with theoretical considerations on the equations formulation, this paper discusses the numerical results based on a model developed in the PROpagation of CORium (PROCOR) platform interfaced with the Open-Calphad thermodynamic software.
\end{abstract}

Keywords: In-vessel corium, solidification, enthalpy, CALPHAD

\section{Introduction}

Within the framework of Severe Accident (SA) analysis in a Light Water Reactor (LWR), following a core meltdown induced by loss of primary coolant (i.e. water), the main aim is to achieve "core coolability" in the reactor containment cavity and eliminate any threats to containment integrity by over-pressurization or melt-through. In this context, the study of corium progression is of prime interest and various reactor design approaches with different SA management strategies have been proposed to contain it. In particular, for "In-Vessel Retention" (IVR), it is the behaviour of the corium in the vessel lower head that determines, through the heat flux distribution on the inner vessel wall, the chances of success of such a strategy (refer to [1, 2]).

Composed of oxide fuel and partially oxidized cladding $\left(\mathrm{UO}_{2}, \mathrm{ZrO}_{2}\right.$ and $\left.\mathrm{Zr}\right)$ as well as structural materials (stainless steel elements $\mathrm{Fe}, \mathrm{Ni}, \mathrm{Cr}$ ), in-vessel corium pool is a complex thermodynamic system that can exhibit various segregated phases at thermodynamic equilibrium. In particular, it can be composed of two immiscible liquid phases (related to a liquid miscibility gap in this thermodynamic system) and the difference in densities results in gravity separation (stratification) of these phases [3]. In the context of IVR where the corium is cooled from the outside, a solid crust can be formed at the interfaces of the different liquid phases (in particular, with the vessel wall) leading to a system exhibiting solid as well as liquid phase segregation simultaneously.

\footnotetext{
${ }^{*}$ Corresponding author

Email address: romain.le-tellier@cea.fr (R. Le Tellier)
} 
With the evolution of temperature and composition, the corium phase distribution changes with time. For instance, when the amount of steel relocated in the corium pool increases, a so-called stratification inversion of the liquid phases may occur. These transient thermochemical phenomena have a strong impact on the phase spatial distribution (including the crust evolution at the boundary of the different liquid phases) and consequently, on the heat flux distribution at the lateral wall through their strong coupling with thermal and thermalhydraulic phenomena. One possible idealized corium pool phase configuration is depicted in Figure 1 for a corium-steel system (U-O-Zr-Steel) as a multi-layer stratified pool.

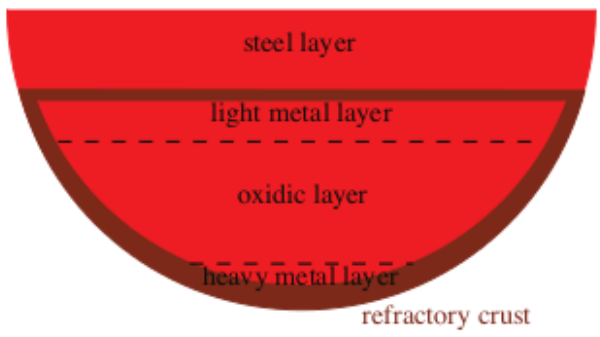

Figure 1: Multilayer corium pool description

Due to complexities involving the coupled thermochemical and thermalhydraulic phenomena at different time and spatial scales and the scarce data available on material properties, the phenomenology involved is not completely understood. In this context, the PROCOR numerical platform [4] developed by the CEA Severe Accidents Laboratory performs sensitivity studies for corium propagation for Light Water Reactors. It is a sufficiently fast tool for performing numerous calculations in a reasonable time frame for statistical studies describing various in-vessel issues.

Thermal modelling of corium pools in PROCOR (as in many integral codes including MAAP [5] and ASTEC [6]) consist of mass and energy conservation equations written for each layer of the corium pool configuration. These energy conservation equations are written in terms of the specific enthalpy of each layer. The evaluation of the heat fluxes at the layer boundaries rely on closures describing the boundary layer heat transfer in terms of heat transfer correlations (refer to [7] for examples). In addition, the models require a choice for formulating the energy conservation equations, depending on the enthalpy-temperature relations. It is to be noted that for multicomponent systems, the dependency of such enthalpy-temperature relations on chemical compositions is important (refer to [8]) and cannot be ignored.

Several benchmark activities between CEA's PROCOR platform and EDF's proprietary versions of MAAP have been performed for comparing the in-vessel corium behaviour [9]. These code cross comparisons have shown that one of the main sources of discrepancies in the results was related to the formulation of energy conservation equations and the associated enthalpy-temperature relations. In particular, it was shown that one important aspect of such enthalpy-temperature relations is their consistency (in terms of the thermodynamic hypotheses and data they rely on) with respect to the corium pool phase segregation modelling. On one hand, the model in MAAP consisting of energy balance equations written in terms of specific enthalpy, used data tabulated in temperature and composition based on the hypothesis that the corium and molten steel species exist as a chemically inactive mixture. However, the boundary condition imposed on the system (i.e. the interface temperature equal to the liquidus temperature of the bulk molten pool) being in accordance with the macro-segregation hypotheses of [10], lead to contradictions between the hypotheses adopted, resulting in erroneous temperature calculations at the poolsolid crust interface. On the other hand, the PROCOR model consisted of energy balance equations written in terms of specific heat capacities with explicit enthalpy-temperature relations. This treatment (consistent with macrosegregation hypotheses) is made possible because the model does not explicitly represent the interface crust (only a fictitious crust is considered in terms of temperature boundary condition for the pool, see [3] for more detail) and the various pools layers are considered to be monophasic. Nevertheless, due to their limiting nature and the requirement of more detailed modelling of the thermochemical interactions in a suboxidized corium-steel system (in particular, the interaction between molten steel and suboxidized corium crust, as per the results of the CORDEB experimental program [11]), the explicit modelling of the crust has been identified as an important objective in PROCOR. Consequently, the same issue of thermodynamic consistency in enthalpy-temperature relations is faced by PROCOR ongoing developments.

In this context, as discussed in [12], it seems interesting to keep a general formulation of energy conservation equations in terms of specific enthalpy and use general enthalpy-temperature relations that can be referred to as an "Equation-of-State" (EOS).

Various thermochemical models involved in in-vessel corium behavior description are based in one way or the other on a thermodynamical description of the corium system using databases obtained with the CALPHAD 
approach [13]. Within this framework, this paper discusses the feasibility and interest of using CALPHAD data for constructing an EOS for in-vessel corium. It is based on a "mock up" model in PROCOR that describes, under a plane solidification front hypothesis, the thermal and thermochemical evolution of the solidifying crust at the boundary of a suboxidized corium pool.

This paper starts with a brief description of the developed integral model (Section 2). For the present study, a ternary system involving the elements U-O-Zr has been considered for a temperature range between [1800, 3000] K. Following the description is a discussion on the numerical results (Section 3) and the assessment of the possibility of extensive and consistent use of CALPHAD database for construction of enthalpy-temperature relations to provide closures to the model.

\section{Model description}

\subsection{Macroscopic conservation equations}

The issue related to the EOS construction has been studied considering the simplified configuration of a suboxidized corium pool with a non-eutectic homogenous composition and a fixed external boundary temperature. This macroscopic model takes into account the thermal and thermochemical evolution of the solidifying corium in terms of use of CALPHAD database for construction of EOS. Figure 2 depicts the two-zone 1-D situation with the liquid corium (denoted by $p$ ) undergoing solidification at its interface (denoted by $\beta$ ) cooled from outside (with the external boundary being denoted by $\alpha$ ). The crust is denoted by $s$ while the interface and external boundary area is $S$.

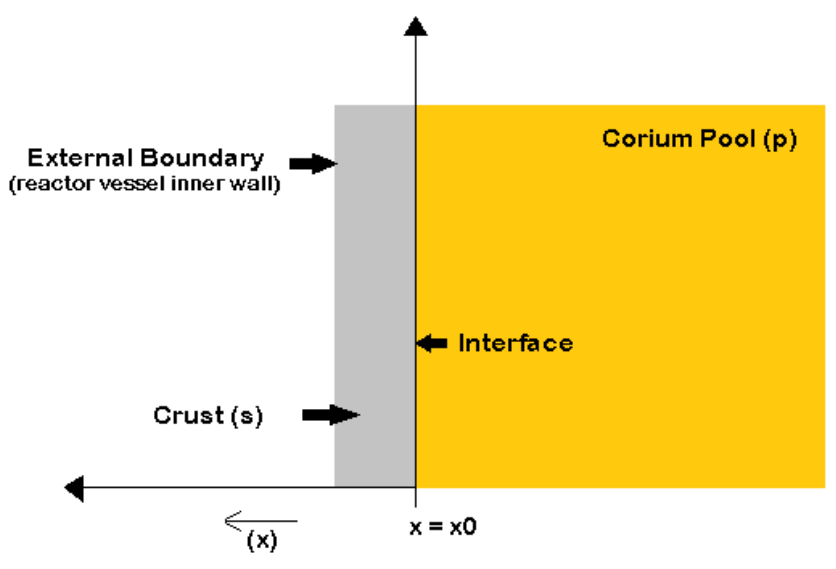

Figure 2: corium-crust configuration : model description

The interface is assumed at the origin $\left(x=x_{0}\right)$ and the crust thickness $\left(x_{s}\right)$ propagates with time till a steady state is achieved. The lumped mass conservation equations for the closed system are written:

$$
\begin{aligned}
& \frac{d m_{p}}{d t}+\dot{m}_{\beta, p}=0 \\
& \frac{d m_{s}}{d t}-\dot{m}_{\beta, p}=0
\end{aligned}
$$

where $m_{p}$ (resp. $m_{s}$ ) refers to the molten pool (resp. crust) mass and $\dot{m}_{\beta, p}$ is the solidification rate at the interface (positive for the mass flow occuring in the direction of the crust from the corium pool).

Applying adiabatic condition on the external boundary for the liquid phase, the energy conservation equations and the interface equation for plane front solidification are written as:

$$
\begin{aligned}
\frac{d}{d t}\left(\bar{h}_{p} m_{p}\right)+\bar{\phi}_{\beta, p} S+\bar{h}_{\beta, p} \dot{m}_{\beta, p} & =\dot{q}_{p}^{\text {mass }} m_{p} \\
\frac{d}{d t}\left(\bar{h}_{s} m_{s}\right)+\bar{\phi}_{\beta, s} S-\bar{h}_{\beta, s} \dot{m}_{\beta, p}+\bar{\phi}_{\alpha, s} S & =\dot{q}_{s}^{\text {mass }} m_{s} \\
\left(\bar{h}_{\beta, p}-\bar{h}_{\beta, s}\right) \dot{m}_{\beta, p}+\left(\bar{\phi}_{\beta, p}+\bar{\phi}_{\beta, s}\right) S & =-\Delta h \dot{m}_{\beta, p}
\end{aligned}
$$

where 
- $\bar{h}_{p}$ and $\bar{h}_{s}$ are the specific enthalpies for the liquid corium and the crust respectively;

- $\bar{h}_{\beta, p}$ and $\bar{h}_{\beta, s}$ are the specific enthalpies for the liquid and solid formed at the interface respectively;

- $\dot{q}_{p}^{\text {mass }}$ and $\dot{q}_{s}^{\text {mass }}$ are the mass power densities for the liquid and solid phases respectively;

- $\bar{\phi}_{\alpha, s}$ is the heat flux at the crust-external boundary interface $(>0)$, directed from the bulk solid towards the external boundary;

- $\bar{\phi}_{\beta, s}$ is the heat flux at the crust-pool interface $(<0)$, directed from the bulk solid towards the interface;

- $\bar{\phi}_{\beta, p}$ is the heat flux at the crust pool interface $(>0)$, directed from bulk corium pool towards the interface;

- $\Delta h \dot{m}_{\beta, p}$ is an additional interfacial term (to be discussed in Section 2.4).

Neglecting mass diffusion in the solid crust (i.e. the diffusive mass flux in the solid at the interface $\left(\bar{J}_{\beta, s}^{j}\right)$ is zero), the species mass conservation and interface equations are:

$$
\begin{aligned}
\frac{d}{d t}\left(\bar{w}_{p}^{j} m_{p}\right)+\bar{J}_{\beta, p}^{j} S+\bar{w}_{\beta, p}^{j} \dot{m}_{\beta, p} & =0 \\
\frac{d}{d t}\left(\bar{w}_{s}^{j} m_{s}\right)-\bar{w}_{\beta, s}^{j} \dot{m}_{\beta, p} & =0 \\
\left(\bar{w}_{\beta, p}^{j}-\bar{w}_{\beta, s}^{j}\right) \dot{m}_{\beta, p}+\bar{J}_{\beta, p}^{j} S & =-\Delta w_{\beta}^{j} \dot{m}_{\beta, p}
\end{aligned}
$$

where

- $\bar{J}_{\beta, p}^{j}$ is the diffusive mass flux for the interfacial liquid composition;

- $\bar{w}_{p}^{j}, \bar{w}_{s}^{j}$ and $\bar{w}_{\beta, s}^{j}$ are the species mass fractions corresponding to the bulk liquid, bulk solid and the interfacial solid composition, respectively

- $\Delta w_{\beta}^{j} \dot{m}_{\beta, p}$ is an additional interface term (to be discussed in Section 2.4 .

For the present case, the mass transfer in the liquid boundary layer is considered to be infinitely fast, such that the bulk composition of liquid is the same as the composition of the liquid present at the interface $\left(\bar{w}_{\beta, p}^{j}=\bar{w}_{p}^{j}\right)$.

The above equations for energy and species conservation include additional interfacial terms, the details of which shall be discussed in Section 2.4, as to the authors knowledge, this has not been discussed thoroughly in the literature. Otherwise, this model is a "standard" plane front solidification model (see, for instance, [14] for analytical solutions in the binary case with a linearized liquidus curve) that has been used in [15] for the interpretation of the LIVE L3A test.

Local thermodynamic equilibrium is assumed to be achieved at the interface such that the interface temperature (denoted by $T_{\beta}$ ) is equal to the liquidus temperature (denoted by $T_{\text {liquidus }}\left(\bar{w}_{\beta, p}^{j}\right)$ ) corresponding to the interface composition of the corium pool (described by $\left(\bar{w}_{\beta, p}^{j}\right)_{j \in S}$, the species mass fractions).

Continuing on a closed system analysis, the heat fluxes at each of the boundaries are modelled. For the interface, the heat transfer from the corium pool to the solid crust is a result of convection and can be expressed using Nusselt-Rayleigh correlation (heat transfer coefficient $h_{\Delta}$ ):

$$
\bar{\phi}_{\beta, p}=h_{\Delta}\left(\bar{T}_{p}-T_{\beta, p}\right)
$$

in terms of the pool average temperature $T_{p}$. In our case, we have used the correlation for upward heat transfer from the BALI experimental program (see [16]).

The heat flux across the solid at the interface as well as the vessel wall boundary is a result of conduction and is expressed in the form of $1 \mathrm{D}$ conduction:

$$
\begin{aligned}
& \bar{\phi}_{\alpha, s} S=-\lambda_{s} S \frac{\partial T_{s}\left(x_{s}, t\right)}{\partial x} \\
& \bar{\phi}_{\beta, S} S=\lambda_{s} S \frac{\partial T_{s}\left(x_{0}, t\right)}{\partial x}
\end{aligned}
$$

with $T_{s}$, the solid temperature distribution and $\lambda_{s}$, the solid thermal conductivity. 
Assuming a quadratic temperature profile to depict the variation across the solid at any time [17] and applying the boundary conditions for crust temperature at the interface $\left(T_{s}\left(x_{0}, t\right)=T_{\beta}\right)$ and the external boundary $\left(T_{s}\left(x_{s}, t\right)=\right.$

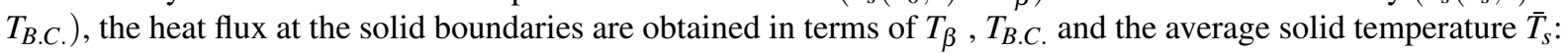

$$
\begin{aligned}
\bar{\phi}_{\alpha, s} & =\lambda_{s} \frac{6 \bar{T}_{s}(t)-2 T_{\beta}-4 T_{B . C .}}{x_{s}} \\
\bar{\phi}_{\beta, s} & =\lambda_{s} \frac{6 \bar{T}_{s}(t)-4 T_{\beta}-2 T_{B . C .}}{x_{s}}
\end{aligned}
$$

Thermophysical properties for both the liquid and solid phases are calculated in PROCOR using TOLBIACICB tabulated species physical properties and mixing laws (see [18]).

\subsection{CALPHAD-based EOS}

The closure of energy conservation equations discussed in Section 2.1 requires enthalpy-temperature relations. While dealing with multicomponent systems, apart from the other state variables like temperature and pressure, it is imperative to consider the dependence of these relations on the chemical composition as well [8]. In the remainder, the pressure possible dependence of this function will be neglected and we will consider that the corium materials density is not affected by pressure. This is a reasonable assumption for corium liquid phases compatible with the Boussinesq approximation of fluid dynamics (see [19]) often used for modeling natural convection in this context.

In this framework, the enthalpy-temperature relation can be described in a general way by the function:

$$
\mathscr{H}: \bar{T},\left(\bar{w}^{j}\right)_{j \in S} \rightarrow \bar{h},\left(\omega_{\gamma},\left(w_{\gamma}^{j}\right)_{j \in S}\right)_{\gamma \in P}
$$

where

- $\bar{T}$ is the average temperature (in $\mathrm{K}$ );

- $\left(\bar{w}^{j}\right)_{j \in S}$ is the average composition defined in terms of mass fraction $\bar{w}^{j}$ for all species $j \in S$;

- $\bar{h}$ is the average mass enthalpy (or specific enthalpy) (in $\mathrm{J} / \mathrm{kg}$ );

- $\left(\omega_{\gamma},\left(w_{\gamma}^{j}\right)_{j \in S}\right)_{\gamma \in P}$ gives the composition of the spatial zone in terms of the different phases: $\omega_{\gamma}$ is the mass fraction of phase $\gamma$ while $\left(w_{\gamma}^{j}\right)_{j \in S}$ gives its composition.

Such relations are referred to as "Equation-Of-State"(EOS) and can be used to obtain a reciprocal temperatureenthalpy relation by solving the non-linear root-finding problem:

$$
\text { find } \bar{T} \text { such that } \mathscr{H}\left[\bar{T},\left(\bar{w}^{j}\right)_{j \in S}\right]=\bar{h}
$$

For a multicomponent, multiphase system, the Gibbs energy can be expressed as a summation of the Gibbs energy functions of all phase contributions:

$$
\mathscr{G}=\sum_{\gamma} m^{\gamma} \mathscr{G}^{\gamma}
$$

where $\mathscr{G}^{\gamma}$ is the specific Gibbs energy (defined as Gibbs energy per unit of mass) for a phase $\gamma$ and the $m_{\gamma}$ represents the mass of the phase. A CALPHAD database consists of Gibbs energy functions for possible phases of a multicomponent system which are in the form of mathematically formulated models, with adjustable parameters. These energy functions depend on state variables like temperature, composition and pressure. They are used for predicting equilibrium conditions for a phase with respect to certain fixed conditions on the state variables and to obtain functions associated with other thermodynamic properties by using the appropriate thermodynamic state relations [13].

For the present study, the U-O-Zr thermodynamic data has been extracted from the NUCLEA'09 database [20]. The temperature that is considered is between $[1800,3000] \mathrm{K}$ and for the composition range of interest, only the liquid phase (LIQUID) and the solid face-centered cubic (U,Zr) $\mathrm{O}_{2-x}$ phase (C1_FCC) have been considered. Both phases are described by a non-ideal associate model based on the following stoichiometric species as constituents: $\mathrm{U}, \mathrm{UO}_{2}, \mathrm{Zr}, \mathrm{ZrO}_{2}$ and $\mathrm{O}$ in such a way that their specific Gibbs energy functions take the form:

$$
\mathscr{G}^{\gamma}: T,\left(w^{j}\right)_{j \in S} \rightarrow g
$$


where $T$ is the temperature $(\mathrm{K})$ and $\left(w^{j}\right)_{j \in S}$ are the mass fraction values for the species constituting the phase $\gamma$.

In order to exploit this CALPHAD data, the CALPHAD numerical tool that has been used is Open-Calphad [21] through an interface in PROCOR (see [22]). The developed solidification model uses this tool for obtaining the local equilibrium conditions at the interface in terms of phase transition temperature $T_{\text {liquidus }}\left(\bar{w}_{\beta, p}^{j}\right)_{j \in S}$ (appearance of the C1_FCC phase) and composition $\left(\bar{w}_{\beta, s}^{j}\right)_{j \in S}$ of the solid C1_FCC phase that is formed upon solidification. In addition, using the fundamental thermodynamic relation $\mathscr{G}=\mathscr{H}-T \mathscr{S}$, enthalpy-temperature relations for both LIQUID and C1_FCC phases have been obtained under the form:

$$
\mathscr{H}^{\gamma}: T,\left(w^{j}\right)_{j \in S} \rightarrow h=\mathscr{G}^{\gamma}\left(T,\left(w^{j}\right)_{j \in S}\right)+T \mathscr{S}^{\gamma}\left(T,\left(w^{j}\right)_{j \in S}\right)
$$

where the specific entropy function is given by:

$$
\mathscr{S}^{\gamma}: T,\left(w^{j}\right)_{j \in S} \rightarrow s=-\left.\frac{\partial \mathscr{G} \gamma}{\partial T}\right|_{\left(w^{j}\right)_{j \in S}}\left(T,\left(w^{j}\right)_{j \in S}\right)
$$

These relations are particular cases of Eq. 14 and have then been used in the energy conservation equations of the solidification model. For the molten pool, assuming that the average liquid temperature $\bar{T}_{p}$ is always greater than the associated liquidus temperature (to ensure that the liquid is monophasic), the closure relations are given by:

$$
\begin{aligned}
\bar{h}_{p}\left(\bar{T}_{p}\right) & =\mathscr{H}^{\text {LIQUID }}\left[\bar{T}_{p},\left(\bar{w}_{p}^{j}\right)_{j \in S}\right] \\
\bar{h}_{\beta, p}\left(T_{\beta}\right) & =\mathscr{H}^{\text {LIqUid }}\left[T_{\beta},\left(\bar{w}_{\beta, p}^{j}\right)_{j \in S}\right]
\end{aligned}
$$

Similarly, for the crust, assuming that its initial state is also a pure C1_FCC phase, the closure relations are given by:

$$
\begin{array}{r}
\bar{h}_{s}\left(\bar{T}_{s}\right)=\mathscr{H}^{\mathrm{C} 1 \_\mathrm{FCC}}\left[\bar{T}_{s},\left(\bar{w}_{s}^{j}\right)_{j \in S}\right] \\
\bar{h}_{\beta, s}\left(T_{\beta}\right)=\mathscr{H}^{\mathrm{C1 \_ FCC}}\left[T_{\beta},\left(\bar{w}_{\beta, s}^{j}\right)_{j \in S}\right]
\end{array}
$$

Note that the initial crust solid structure is dependent on the initial crust formation transient and the above assumption would not be compatible with quenching for instance. However, this modelling aspect is out of the scope of this paper.

\subsection{Numerical coupling scheme for time integration}

The time integration of system conservation equations coupled with CALPHAD related properties requires numerical discretization for solving the corium solidification transient. To do so, a macro time scale is defined with prescribed time-step $\Delta t$ and a semi-explicit coupling between mass/energy conservation equations and species mass balance is considered. Over a macro time-step $[t, t+\Delta t$, material compositions are considered to be constant and equal to their values at $t$ in such a way that the interface temperature along with the enthalpy-temperature relations of Eqs. 15, 16, 17 and 18 can be evaluated at $t$. Then, an explicit Euler scheme is used to integrate the mass and energy conservation equations for the crust (Eqs. 2] and 5) and corium (Eqs. 2] and 4) from $t$ to $t+\Delta t$ over a micro time-step grid (with a micro time-step $\delta t$ ). Finally, the updated phase compositions at $t+\Delta t$ are obtained by time integration of the species conservation equation Eqs. 7 and 8 using the explicit scheme approximation. For instance, for the solid phase, Eq. 8 gives:

$$
\left.\left(\bar{w}_{s}^{j} m_{s}\right)\right|_{t+\Delta t}=\left.\bar{w}_{\beta, s}^{j}\right|_{t}\left[\left.\left(\dot{m}_{\beta, p}\right)\right|_{t+\Delta t}-\left.\left(\dot{m}_{\beta, p}\right)\right|_{t}\right] \Delta t+\left.\left(\bar{w}_{s}^{j} m_{s}\right)\right|_{t}
$$

These updated system compositions act as inputs to the model for the next macro time-step.

\subsection{Redox reaction at the interface}

The interfacial terms of Eqs. 5 and 8 introduced in Section 2.1 take into account the fact that, in the general case, the species mass conservation may no longer hold true due to a possible formation of different species in the solidified crust at the interface from those present in the interfacial liquid. For the present study, in case of suboxidized corium, the NUCLEA database predicted a difference in the composition of the solid formed at thermodynamic equilibrium at the liquidus temperature from the interfacial liquid composition. In particular, appearance of non-zero mass fraction of $U$ species in the solid was noted. This presence may be interpreted as 
a result of the redox reaction $\left(\mathrm{UO}_{2}+\mathrm{Zr} \rightleftharpoons \mathrm{U}+\mathrm{ZrO}_{2}\right)$ occurring in direction of formation of $\mathrm{U}$ and $\mathrm{ZrO}_{2}$ in the interfacial liquid. The extra interfacial terms $-\Delta h \dot{m}_{\beta, p}$ and $-\Delta w_{\beta}^{j} \dot{m}_{\beta, p}$ are there to correct the balance equations Eqs. 5 and 8 accordingly.

The non-zero mass fraction for $U$ species relative to the associate model of the C1_FCC phase corresponds to the slight sub-stoichiometry of the $(\mathrm{U}, \mathrm{Zr}) \mathrm{O}_{2-x}$ phase. This sub-stoichiometry is to be related to vacancies in the face-centered cubic lattice and not be confused with appearance of an actual metallic U phase.

A quantity $r$ was defined, which denotes the mass of interfacial liquid required for solidifying a unit mass of solid at equilibrium composition. Out of this mass $r$ of the initial liquid at the interface $\left(\bar{w}_{\beta, p}^{j}\right)_{j \in S}$, a unit mass of solid $\left(\bar{w}_{\beta, s}^{j}\right)_{j \in S}$ is formed, leaving $(r-1)$ amount of "residual" liquid of composition $\left(\bar{w}_{\beta, p^{\prime}}^{j}\right)_{j \in S}$. From there, the overall species mass variation occurring at the interface gives us the value of $\Delta w_{\beta}^{j}$ as:

$$
\Delta w_{\beta}^{j}=\bar{w}_{\beta, s}^{j}+(r-1) \bar{w}_{\beta, p^{\prime}}^{j}-r \bar{w}_{\beta, p}^{j}(j \in S)
$$

In practice, the value of $r$ can be calculated in a general way by :

$$
r=\max _{i \in E}\left(\frac{\bar{e}_{\beta, s}^{i}}{\bar{e}_{\beta, p}^{i}}\right)
$$

where $\left(\bar{e}_{\beta, s}^{i}\right)_{i \in E}$ (resp. $\left.\left(\bar{e}_{\beta, p}^{i}\right)_{i \in E}\right)$ are the component mass fractions associated with the species mass fractions $\left(\bar{w}_{\beta, s}^{j}\right)_{j \in S}\left(\operatorname{resp} .\left(\bar{w}_{\beta, p}^{j}\right)_{j \in S}\right)$.

In our case of interest, the "limiting "element for the solidification of in the interfacial liquid is uranium in such a way that $r=\frac{\bar{e}_{\beta, s}^{U}}{\bar{e}_{\beta, p}^{U}}$.

Following this, the "residual "liquid composition in terms of species mass fraction $\left(\bar{w}_{\beta, p^{\prime}}^{j}\right)_{j \in S}$ can be obtained from a simple element mass conservation and a subsequent hypothesis assuming that the "residual "liquid is obtained from a local equilibrium calculation at the interface temperature $\left(T_{\beta}\left(\bar{w}_{\beta, p}^{j}\right)_{j \in S}\right)$. Note that this is the same assumption that has been used in the phase-field model of the multiphase multicomponent diffusion for in-vessel corium in [23].

In this context, $\Delta h$ is interpreted as the specific enthalpy of reaction associated to the oxygen redistribution in the interfacial liquid concurrent to solidification and it is evaluated as:

$$
\Delta h=r\left(\mathscr{H}^{\text {LIQUID }}\left[T_{\beta},\left(\frac{1}{r} \bar{w}_{\beta, s}^{j}+\left(1-\frac{1}{r}\right) \bar{w}_{\beta, p^{\prime}}^{j}\right)_{j \in S}\right]-\bar{h}_{\beta, p}\right)
$$

\section{Numerical results and analysis}

The developed model was subjected to initial verification tests, with respect to global energy balance and the analysis of the heat conduction profile in the solid crust at steady state. For the sake of conciseness, such tests are not reported here. Following this, the model was used for evaluation of the principal variables of interest on two solidification transients for suboxidized and fully oxidized corium systems. These systems had a common $\mathrm{U} / \mathrm{Zr}$ molar ratio $\left(R_{U / Z r}\right)$ of 1.2 and differ in the $\mathrm{Zr}$ molar oxidation degree $C_{Z r}$ being $30 \%$ and $100 \%$ respectively. For the initial system conditions of the two cases (refer to Table 1 ), the evolution of different parameters were studied for a macro (resp. micro) time-step size of $\Delta t=100 \mathrm{~s}$ (resp. $\delta t=1 \mathrm{~s}$ ) and have been summarized below. These conditions have been selected in such a way that a monotonic solidification transient is obtained for both corium configurations. The transient calculations are performed until steady state (defined by a criterion relative to the crust mass variation between two consecutive time-steps that should be lower than $10^{-4} \mathrm{~kg}$ ). 


\begin{tabular}{|c|c|c|}
\hline Parameters & $C_{Z r}=30 \%$ & $C_{Z r}=100 \%$ \\
\hline Solid initial mass $m_{s}(\mathrm{~kg})$ & 1000 & 1000 \\
\hline Liquid initial mass $m_{p}(\mathrm{~kg})$ & 20000 & 15000 \\
\hline Solid initial temperature $\bar{T}_{s}(\mathrm{~K})$ & 2200 & 2500 \\
\hline Liquid initial temperature $\bar{T}_{p}(\mathrm{~K})$ & 2700 & 2900 \\
\hline Mass power density $\dot{q}^{\text {mass }}(\mathrm{W} / \mathrm{kg})$ & 100 & 100 \\
\hline Initial interface temperature $T_{\text {liquidus }}\left(\bar{w}_{\beta, p}^{j}(t=0)\right)(\mathrm{K})$ & 2637.0 & 2874.7 \\
\hline External boundary temperature $T_{B . C .}(\mathrm{K})$ & 1800 & 1800 \\
\hline Interface surface area $S(\mathrm{~m})$ & 12.56 & 12.56 \\
\hline
\end{tabular}

Table 1: Initial inputs to the thermal model: Case $C_{Z r}=30 \%$ and $C_{Z r}=100 \%$

The evolution of temperature for the solid and liquid (bulk as well as at the interface) phases of the two systems was observed to be different, with the change in the liquidus temperature being small for the completely oxidized corium system $\left(C_{Z r}=100 \%\right)$ compared to that for the suboxidized corium system $\left(C_{Z r}=30 \%\right)$ (refer to Figure 3 . This difference in trends is due to the dependence of the liquidus temperature on the interface composition. For a completely oxidized system, the change in composition is observed to be small, with the maximum variation being recorded for the mass fraction of $\mathrm{ZrO}_{2}$ in the solid crust $(\approx-12 \%)$ as well as in the bulk liquid $(\approx-2.3 \%)$, resulting in almost no variation of the liquidus temperature $\left(<-4^{\circ}\right)$. On the other hand, the suboxidized corium undergoes larger changes in its composition, the highest being recorded for the amount of $\mathrm{Zr}$ present in the solid crust $(\approx-64 \%)$ and the appearance of species $U$ in the solid $\left(\bar{w}_{s}^{U}=0.062\right)$ (see Section 2.4. The larger changes in the system composition result in a more significant variation of the liquidus temperature $\left(\approx-59^{\circ}\right)$.

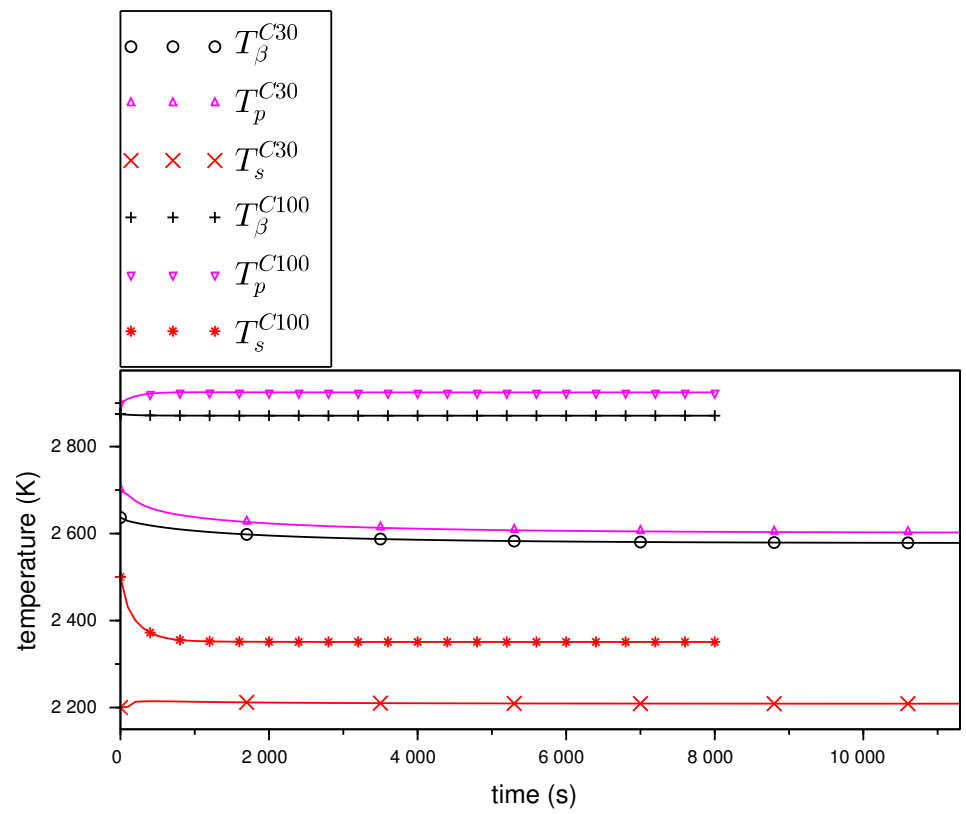

Figure 3: time evolution of the bulk and interface temperatures - comparing cases: $C_{Z r}=30 \%$ and $C_{Z r}=100 \%$

Apart from the changes in the temperature and composition of the phases, the redox reaction also has an impact on the phase change enthalpy values for the corium composition. As shown in Section 2.4, the redox reaction results in addition of a term $(\Delta h)$ in the interface energy balance equation. The value of $\Delta h$ was computed at every macro time-step and it was observed to be small compared to the phase change enthalpy values $\left(\bar{h}_{\beta, p}-\bar{h}_{\beta, s}\right)$ to cause any significant changes on the solidification transient (refer to Figure 4). Note that the value of $\Delta h$ for the completely oxidized corium is not strictly zero (at $t=0, \Delta h \approx 0.5 \%\left(\bar{h}_{\beta, p}-\bar{h}_{\beta, s}\right)$ ); this is related to the solid composition calculated by Open-Calphad that exhibits very small amounts of $\mathrm{U}\left(\approx 10^{-5}\right)$ and $\mathrm{O}\left(\approx 10^{-6}\right)$ for $C_{Z r}=100 \%$, which can be attributed to the numerical convergence of the minimization process and has no impact on the overall transient. 


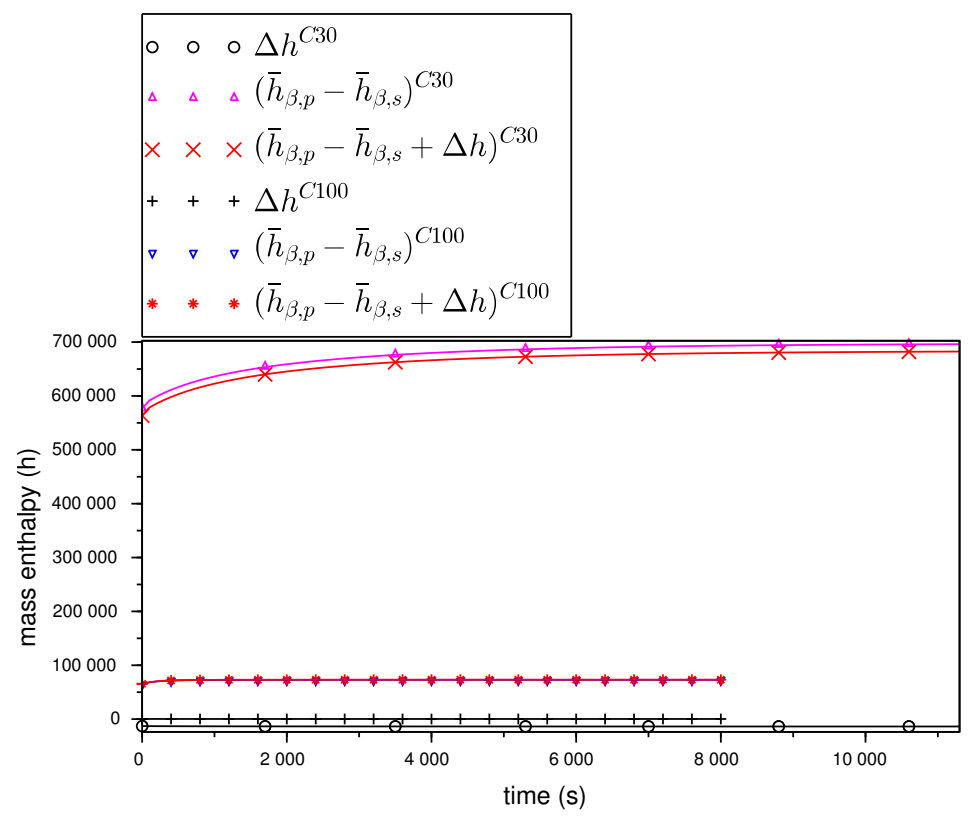

Figure 4: time evolution of the phase change enthalpies - comparing cases: $C_{Z r}=30 \%$ and $C_{Z r}=100 \%$

The large difference in the phase change enthalpy values (neglecting the additional interface term) for $C_{Z r}=$ $30 \%$ and $C_{Z r}=100 \%$ cases (with $\left(\bar{h}_{\beta, p}-\bar{h}_{\beta, s}\right)$ for $C_{Z r}=30 \% \approx 10\left(\bar{h}_{\beta, p}-\bar{h}_{\beta, s}\right)$ for $C_{Z r}=100 \%$ at $t=0$ ) (Figure 4) is mainly related to the modification in the enthalpy value for the liquid phase. At $t=0, C_{Z r}=30 \%$ value for $\bar{h}_{\beta, p}$ is $32 \%$ higher than $C_{Z r}=100 \%$ value while for the solid this increase is limited to $14 \%$. This important variation is attributed to the large dependence of the enthalpy as a function of the oxygen content. Note that for pure compounds such as $\mathrm{UO}_{2}$ and $\mathrm{ZrO}_{2}$, it has been verified that this dispersion of specific latent heat of solidification is largely reduced and values are consistent with published data as shown in Table 2 . The liquid enthalpy values computed from CALPHAD data are likely to have a large uncertainty because of the scarcity of associated experimental data and measurements of latent heat of solidification for such ternary systems at high temperature are not available [24].

\begin{tabular}{|c|c|c|c|}
\hline compound & NUCLEA'09 & {$[25]$} & {$[26]$} \\
\hline $\mathrm{UO}_{2}$ & 276 & 277 & $259 \pm 15$ \\
\hline $\mathrm{ZrO}_{2}$ & 730 & 706 & n/a \\
\hline
\end{tabular}

Table 2: Latent heat of fusion in $\mathrm{kJ} / \mathrm{kg}$ for $\mathrm{UO}_{2}$ and $\mathrm{ZrO}_{2}$ from different sources

Because of the considerable difference in the values of phase change enthalpies $\left(\bar{h}_{\beta, p}-\bar{h}_{\beta, s}+\Delta h\right)$ between the $C_{Z r}=30 \%$ and $C_{Z r}=100 \%$ cases, an important difference in the corium solidification rate is observed. This difference increases with time, resulting in a slower solidification rate for $C_{Z r}=30 \%$ compared to $C_{Z r}=100 \%$. Figure 5 depicts the time evolution of crust mass for both cases. It can be observed that the suboxidized corium system takes a much longer time than the completely oxidized corium system to reach the steady state owing to its decreasing solidification rate.

A parametric study on the explicit time-step integration was performed for the $C_{Z r}=30 \%$ case to check the accuracy and stability of the integration scheme used. The results obtained with a step-size value smaller (i.e. $\Delta t=10 \mathrm{~s}$ ) than the initially chosen value of $\Delta t=100 \mathrm{~s}$ were very similar to each other. For a larger time-step $(\Delta t=1000 \mathrm{~s})$, limited damped oscillations are observed on the crust mass with the maximum amplitude observed at the end of first macro time-step (updated crust mass value at $t=1000^{-} \mathrm{s}$ being approximately $2.5 \%$ less than the crust mass evaluated at $t=1000^{+} s$ ) and the differences decreasing for subsequent time-steps. This can be explained as it was observed that for a larger time-step, the value of heat fluxes at the interface $\bar{\phi}_{\beta, p}$ and $\bar{\phi}_{\beta, s}$ underwent oscillations due to the interface temperature discontinuities (due to the composition update at the end of each macro time-step) that lead to changes of sign of $\left(\bar{\phi}_{\beta, p}+\bar{\phi}_{\beta, s}\right)$ before and after the composition updates. 


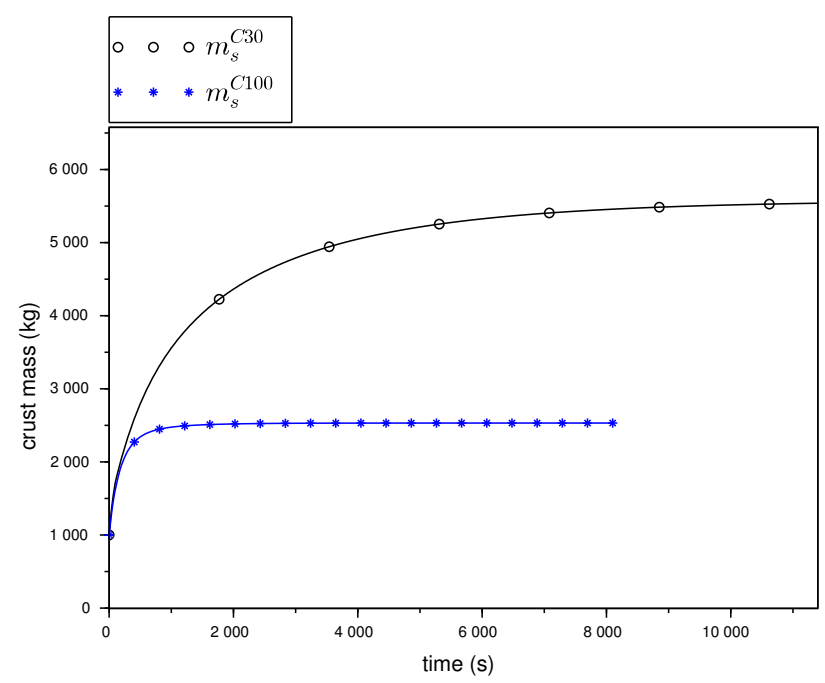

Figure 5: time evolution of crust mass evolution - comparing cases: $C_{Z r}=30 \%$ and $C_{Z r}=100 \%$

Finally, the validity of using $\mathscr{H}^{\text {LIQUID }}$ and $\mathscr{H}^{\text {C1_FCC }}$ functions as EOS in the conservation equations is, in the general case, related to the linearity of these functions with respect to temperature and composition. It is known that these EOS are useful in predicting the system behaviour at a microscopic level but their validity at macroscopic levels need to be tested as well. This is because at a microscopic level, the specific enthalpy can be expressed as a function of the local temperature and composition as $\mathscr{H}^{\gamma}\left(T,\left(w^{j}\right)_{j \in S}\right)$ for a given phase $\gamma$. However, this relation may not be valid for corresponding mass averaged enthalpy $\bar{h} m=\int_{\Omega} \rho h d V$ where $\Omega$ refers to the spatial zone in which volume averaging is applied and $m=\int_{\Omega} \rho d V$ is the mass of the spatial zone. This can be explained from the fact that average of product of two quantities is not always same as the product of the averages [8].

In order to test the validity of the EOS, the variation of the specific enthalpy as a function of temperature is depicted in Figure 7(resp. Figure 6) for the solid (resp.liquid) phase at initial and steady state compositions over the temperature range $\left[T_{B . C .}, T_{\beta}\right]$ (resp. $\left[T_{\beta}, T_{p}\right]$ ) for the $C_{Z r}=30 \%$ case. In addition, the dependence of both liquid and solid specific enthalpies on the phase composition at a fixed temperature was obtained (see Figure 8). The composition was varied as a linear combination of the initial and final compositions $\left(\bar{w}_{0}^{j}\right.$ and $\left.\bar{w}_{\infty}^{j}\right)$ as $\left(\bar{w}^{j}\right)_{j \in S}=$ $\left((1-\gamma) \bar{w}_{0}^{j}+\gamma \bar{w}_{\infty}^{j}\right)_{j \in S}$ with parameter $\gamma \in[0,1]$. It is verified a posteriori that these functions are almost linear in the range of temperatures and compositions covered by the transient. The maximum standard deviation of the residuals with respect to a linear regression is about $4406 \mathrm{~J} / \mathrm{kg}(<0.2 \%)$ recorded for the value of $\bar{h}_{s}$ for its variation with temperature at a fixed initial composition.

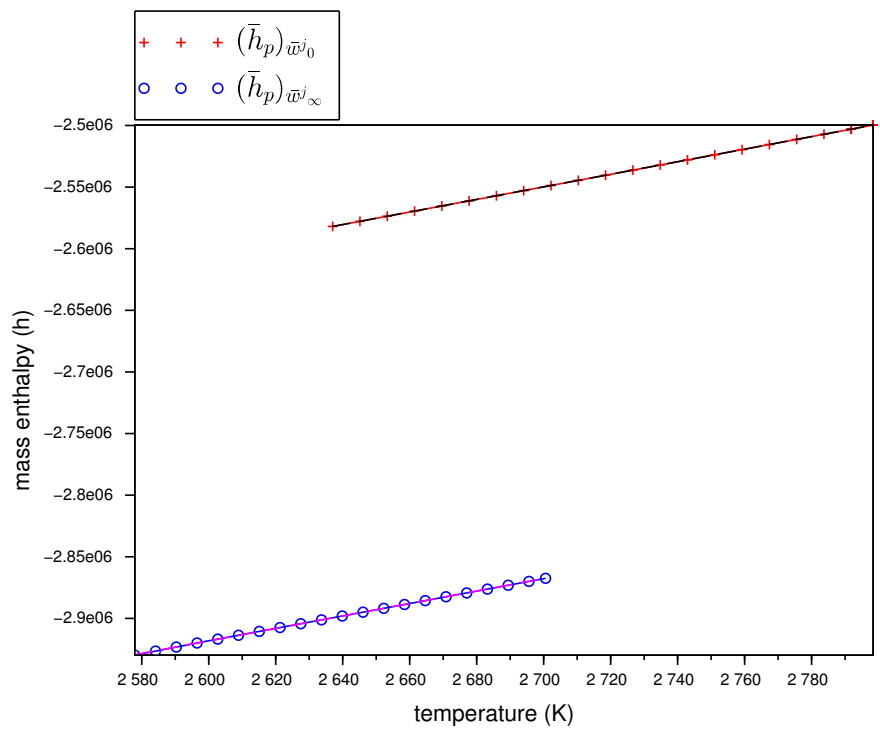

Figure 6: variation of $\mathscr{H}_{\text {LIQUID }}\left[T,\left(\bar{w}^{j}\right)_{j \in S}\right]$ with temperature, for a fixed composition 


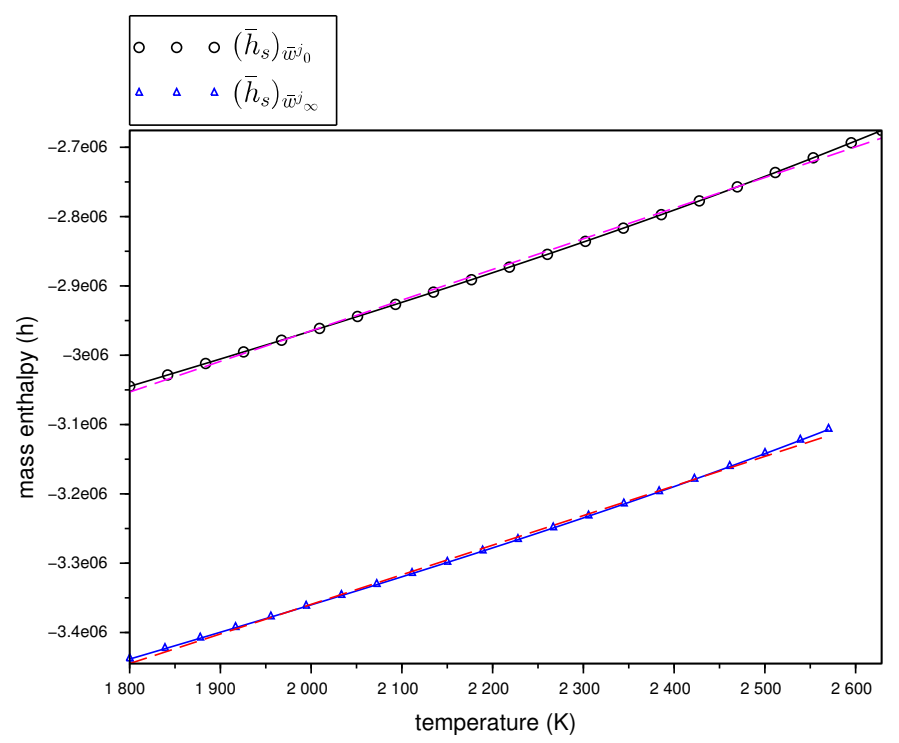

Figure 7: variation of $\mathscr{H}_{\mathrm{C1} \text { _FCC }}\left[T,\left(\bar{w}^{j}\right)_{j \in S}\right]$ with temperature, for a fixed composition

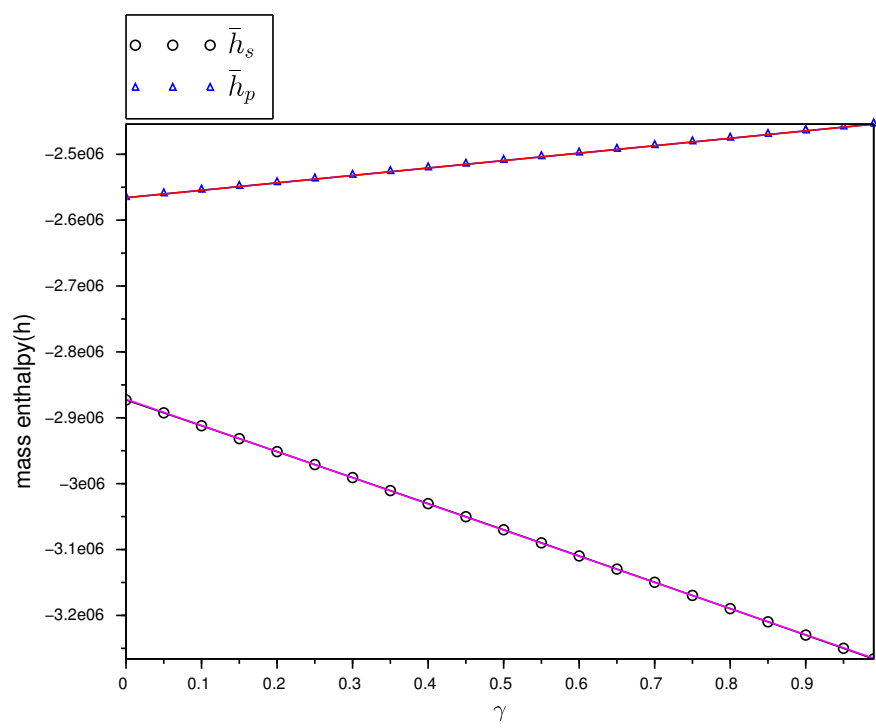

Figure 8: variation of $\mathscr{H}\left[T,\left(\bar{w}^{j}\right)_{j \in S}\right]$ with composition, for a fixed temperature

\section{Conclusion and future prospectives}

This paper has presented a corium solidification model for the ternary U-O-Zr system based on the extensive use of U-O-Zr CALPHAD data. The approach has allowed us to discuss the possibility of using CALPHAD database for constructing EOS to be used as closures in corium-related thermal models associated with phase segregation. In particular, it was shown that the consistent use of CALPHAD data for evaluating interface quantities related to the local equilibrium assumption requires a modification of the species conservation equations for a suboxidized corium. The model was tested for consistency and the results obtained have been reported. They have confirmed the feasibility of using the CALPHAD database to obtain thermodynamically consistent closures for the conservation equations. However, it is to be noted that this model is based on the assumption that both solid and liquid compositions are monophasic and a possible solid phase fraction in the bulk liquid layer has not been considered. Moreover, the temperature and composition range covered in the model is limited, thus making the construction of EOS simpler than in the general case. Future considerations involving points like finite diffusion into the liquid boundary layer and the presence of solid fractions in the bulk liquid will require further modifications in the model as well as more extensive strategies regarding the interpolation of the CALPHAD-based thermodynamic properties. 


\section{Acknowledgments}

This work has been carried out within the framework of the PROCOR platform development funded by CEA, EDF and AREVA.

\section{References}

[1] J. L. Rempe, D. L. Knudson, M. Cebull, C. L. Atwood, Potential for in-vessel retention through ex- vessel flooding, in: Proc. of the OECD/CSNI Workshop on In-Vessel Core Debris Retention and Coolability, 1998.

[2] T. G. Theofanous, C. Liu, S. Addition, S. Angelini, O. Kym al ainen, T. Salimassi, In-vessel coolability and retention of a core melt, Nuclear Engineering and Design 169 (1997) 1-48.

[3] R. Le Tellier, L. Saas, S. Bajard, Transient stratification modelling of a corium pool in a LWR vessel lower head, Nuclear Engineering and Design 287 (2015) 68-77.

[4] R. Le Tellier, L. Saas, F. Payot, Phenomenological analyses of corium propagation in LWRs: the PROCOR software platform, in: Proc. of the 7th European Review Meeting on Severe Accident Research ERMSAR2015, Marseille, France, 2015.

[5] Fauske \& Associates Inc., MAAP5 - modular accident analysis program for LWR power plants, in: Code Structure and Theory, Vol. 2, Electric Power Research Institute, 2008, Ch. 3.

[6] P. Chatelard, N. Reinke, S. Arndt, S. Belon, L. Cantrel, L. Carenini, K. Chevalier-Jabet, F. Cousin, J. Eckel, F. Jacq, C. Marchetto, C. Mun, L. Piar, $\{$ ASTEC $\}\{$ V2 $\}$ severe accident integral code main features, current v2.0 modelling status, perspectives, Nuclear Engineering and Design 272 (2014) 119 - 135. doi:http: //dx.doi.org/10.1016/j.nucengdes.2013.06.040

[7] L. Zhang, Y. Zhou, Y. Zhang, W. Tian, S. Qiu, G. Su, Natural convection heat transfer in corium pools: A review work of experimental studies, Progress in Nuclear Energy 79 (2015) 167-181. doi:10.1016/j. pnucene.2014.11.021.

[8] M. Schneider, C. Beckermann, Effects of simplified enthalpy relations on the prediction of heat transfer during solidification of a lead-tin alloy, Applied Mathematical Modelling 15 (11-12) (1991) 596-605. doi: 10.1016/S0307-904X(09)81005-6.

[9] N. Bakouta, R. Le Tellier, L. Saas, Assessment of advanced corium-in-lower-head models in MAAP and PROCOR codes, in: Proc. of the 7th European Review Meeting on Severe Accident Research ERMSAR, Marseille (France), 2015.

[10] J. M. Seiler, A. Fouquet, K. Froment, F. Defoort, Theoretical analysis for corium pool with miscibility gap, Nuclear Technology 141 (2003) 233-243.

[11] D. Jacquemain, D. Vola, R. Meignen, J. M. Bonnet, F. Fichot, E. Raimond, M. Barrachin, Past and future R\&D at IRSN on corium progression and related mitigation strategies in a severe accident, in: Proc of the 16th International Topical Meeting on Nuclear Reactor Thermalhydraulics. NURETH-16, Chicago, USA, 2015 .

[12] P. Despret, J.-L. Dulong, P. Villon, The heat equation strictly written in enthalpy, in: Proc. of CSMA 2015 (12me Colloque National en Calcul des Structures), 2015.

[13] H. Lukas, S. G. Fries, B. Sundman, Computational Thermodynamics: The Calphad Method, Cambridge University Press, 2007.

[14] H. E. Huppert, M. G. Worster, Dynamic solidification of a binary melt, Nature 314 (6013) (1985) 703-707. doi:10.1038/314703a0.

[15] Q. T. Pham, J. M. Seiler, H. Combeau, X. Gaus-Liu, F. Kretzschmar, A. Miassoedov, Modeling of heat transfer and solidification in LIVE L3a experiment, International Journal of Heat and Mass Transfer 58 (1-2) (2013) 691-701. doi:10.1016/j.ijheatmasstransfer.2012.11.030.

[16] J. M. Bonnet, J. M. Seiler, Thermohydraulic phenomena in corium pool: the bali experiment, in: Proc. of ICONE 7, Tokyo, Japan, 1999. 
[17] R. Le Tellier, E. Skrzypek, L. Saas, On the treatment of plane fusion front in lumped parameter thermal models with convection, Applied Thermal Engineering submitted in October 2016.

[18] B. Spindler, B. Tourniaire, J. M. Seiler, Simulation of MCCI with the TOLBIAC-ICB code based on the phase segregation model, Nuclear Engineering and Design 236 (2006) 2264-2270.

[19] R. N. Hills, P. H. Roberts, On the motion of a fluid that is incompressible in a generalized sense and its relationship to the boussinesq approximation, Stability and Applied Analysis of Continuous Media 1 (3) (1991) 205-212.

[20] B. Cheynet, P. Chevalier, E. Fischer, Thermosuite, Calphad 26 (2) (2002) 167-174.

[21] B. Sundman, U. R. Kattner, M. Palumbo, S. G. Fries, OpenCalphad - a free thermodynamic software, Integrating Materials and Manufacturing Innovation 4 (1). doi:10.1186/s40192-014-0029-1.

[22] B. Sundman, U. R. Kattner, C. Sigli, M. Stratmann, R. Le Tellier, M. Palumbo, S. G. Fries, The OpenCalphad thermodynamic software interface, Computational Materials Science 125 (2016) 188-196.

[23] C. Cardon, R. Le Tellier, M. Plapp, Modelling of liquid phase segregation in the uranium-oxygen binary system, CALPHAD: Computer Coupling of Phase Diagrams and Thermochemistry 52 (2016) 47-56.

[24] C. Guéneau, private communication, CEA Saclay, DEN/DANS/DPC/SCCME (October 2016).

[25] N. I. Kolev, Thermo-physical properties for severe accident analysis, Springer Berlin Heidelberg, Berlin, Heidelberg, 2012, pp. 617-807.

[26] IAEA (Ed.), Thermophysical Properties of Materials for Nuclear Engineering: A Tutorial and Collection of Data, IAEA, 2008. 